\title{
Configurations of Causal Conditions Leading to Geotagged Image and Social Context Recognition in the Case of AI Media Platform
}

\author{
Adrian Micu \\ adrian.micu@ugal.ro \\ Alexandru Capatina \\ alexandru.capatina@,ugal.ro \\ Dunarea de Jos University of Galati, Romania \\ Angela-Eliza Micu \\ angelaelizamicu@yahoo.com \\ Ovidius University of Constanta, Romania \\ Robert Rusu \\ Constantin Avram \\ Dunarea de Jos University of Galati, Romania
}

\begin{abstract}
The increasing use of geotagged image and social context recognition in Social Media Marketing offer companies that are looking for using emerging AI Media solutions opportunities to post relevant content on each social network in order to drive the maximum engagement with their audience. This research aims to identify and further analyze the feedback of potential users of an AI-based platform for Social Media Marketing, regarding its capabilities focused on geotagged image and social context recognition. Through this hybrid quantitative-qualitative approach, marketing professionals from FutureWeb research team propose different causal configurations of forthcoming capabilities of the AI-based platform. Gaining visibility through brand related photo or video geotagged on social media; free advertising through tagging a location while people add an image containing a brand's logo; incentives given to geotagged images uploaded on social networks; richer behavioural data provided by geotagged image recognition features and capitalizing on location-based image recognition to predict consumer behaviour and improve services are analysed as antecedents of intention to test AI Media platform.
\end{abstract}

Keywords: Artificial Intelligence, geotagging, social recognition, social media

\section{Introduction}

Bridging geotagged image and social context recognition with other relevant features related to image recognition in social networks, this research framework proposes causal configuration of five antecedent conditions, exploring recipes of building a prototype of AI Media platform.

Most research papers focused on the users' intention of testing platform assume that relations among the variables explaining the capabilities proposed for the forthcoming IT platform are symmetric and offer one single best solution explaining the outcome. To address this gap in the literature, this paper proposes a configurational model in the attempt to identify the different causal patterns of factors that influence potential users' intentions to test an AI media platform, the main outcome of FutureWeb project. We explore the following research question: What configurations of antecedent conditions, grouped in five core areas of Social Media Marketing (Visibility, Advertising, Incentives, Behaviour, Predictions) lead to high and low/medium intentions to test the AI Media platform? 


\section{Theoretical background}

Social networks are gaining popularity for sharing photos, videos and locations where they have been taken or recorded. Researchers as Ivanov et al. (2012) have developed an original platform for automatic geotag sharing in social media by associating locations with specific landmarks and using object duplicate detection for tag sharing, without enabling social context recognition. Hays and Efros (2008) are among the first researchers who identified and addressed the challenge of estimating the location of an image shared on social networks using only its visual content, based on a sample of geotagged Flickr images. Using a predefined set of visual features, they used nearest-neighbour search to locate the image in line with the reference set.

Considering that geotags have huge potential to improve performance of visual image recognition, Yaegashi and Yanai (2010) propose the introduction of Multiple Kernel Learning (MKL) into geotagged image recognition to estimate the contribution weights of visual features of photo images, extremely useful in social media posts. Without pretending the recognition of social context of images posted on social networks, a group of researchers coordinated by Knopp developed a method for automatic detection of geotagged images and spatially localized groups of confusing features, proving that suppressing them lead to improvement of location recognition performance (Knopp et all., 2010). To cope with the challenges and opportunities in working with geotagged images in social networks, we are witnesses of an increasing demand for large standardized geotagged datasets in the social media research community (Choi et al., 2014).

A solution to integrate social context recognition with geotagged image recognition is offered by an invention (Harris et al., 2014) that promotes geo-feed based on a specification of one or more geo-locations, using parameters such as content providers to include, types of content to include, date ranges, content matching patterns, keywords, and/or other parameters that instruct the system as to which content should be included in the geo-feed module.

In the last years, an increased emphasis on modeling and using contextual geotagged image and information has been observed within multimedia understanding and computer vision domains (Tsai et al., 2005; Luo and al.,2011). Contextual modeling often gives an opportunity to derive more insights from images and data related to a geo-location. A few sources of contextual recognition of images shared in social networks are: meta-data captured with pictures or videos, relationships between spatio-temporal segments in multimedia data (Luo et al., 2006), or patterns in multimedia collections as a whole (Yuan et al., 2008). Geographic information has been reconsidered by multimedia and vision researchers in tailored contextual modeling frameworks.

Most research studies on geolocalizing images posted on social networks takes one of two specific approaches. The first is to design discrete models using emerging technologies for a set of specific places, like buildings and other landmarks, and then to match unlabeled images against these models (Lee et al, 2015). The second approach views geo-localization as an image matching problem: compare a query image to a large set of reference images having known GPS coordinates, and estimate a geo-tag based on the visual matches (Hays and Efros, 2008).

\section{Method and measurements}

Fuzzy-sets Qualitative Comparative Analysis (fsQCA) methodology has been considered appropriate in order to explore the intention to test a future Artificial Intelligence-based platform; the objective is to understand how causal configurations of geotagged image and social context recognition capabilities proposed for the future AI Media software (integrated in Image analysis, Audience analysis and Sentiment analysis) affect to the outcome. Research sample involves 25 cases (respondents answers to six items - presented in Appendix, the first five items representing antecedents, while the sixth being the outcome). The research framework (Figure 1) has been built in line with the fsQCA requirement to design combinations of causally relevant conditions (Ragin and Fiss, 2008). This method relies on the application of Boolean algebra to identify causal combinations of antecedent conditions (gaining visibility through brand related photo or video geotagged on social media; free advertising through tagging a location while people add an 
image containing a brand's logo; incentives given to geotagged images uploaded on social networks; richer behavioural data provided by geotagged image recognition features and capitalizing on location-based image recognition to predict consumer behaviour and improve services) that lead to the outcome (high/low intention to test AI Media platform capabilities).

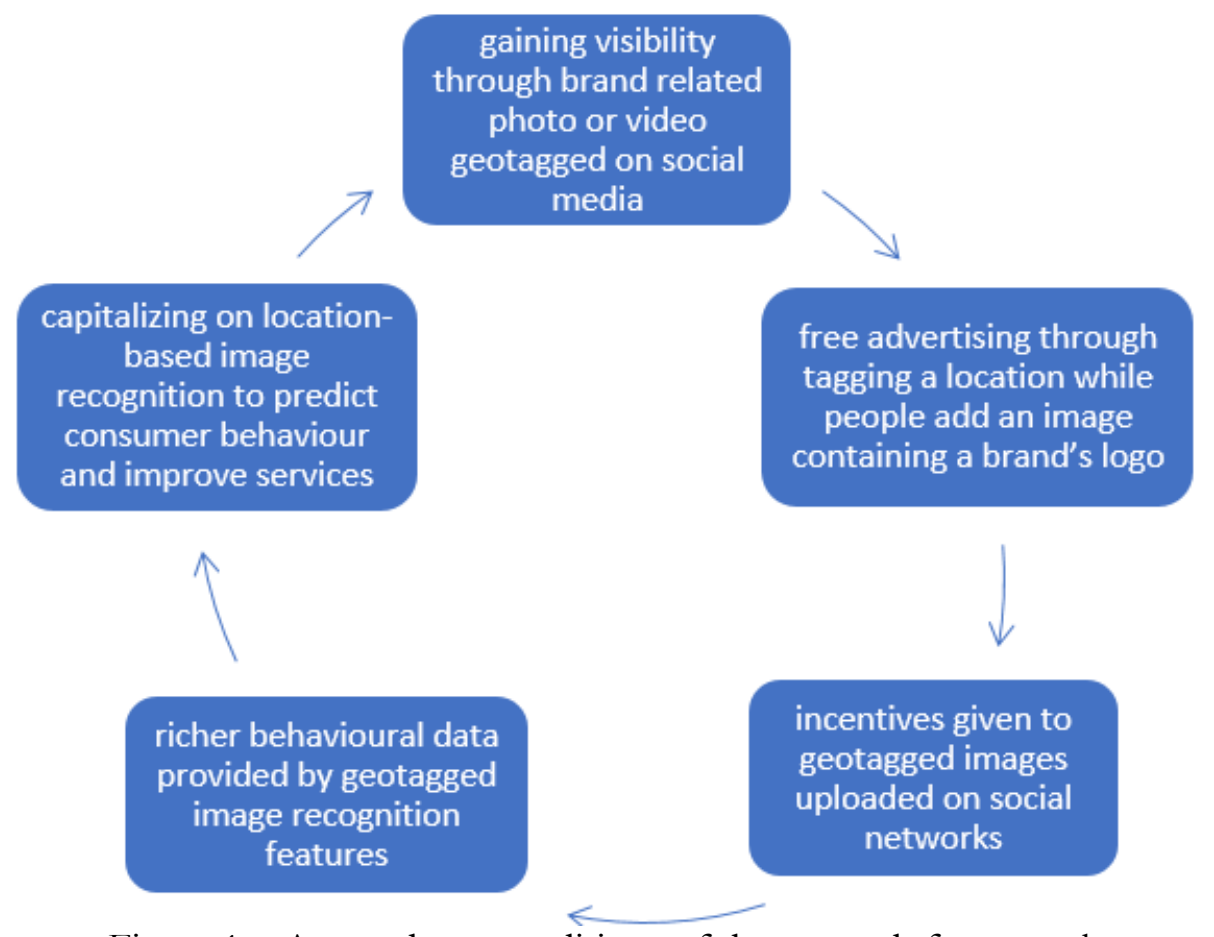

Figure 1 - Antecedents conditions of the research framework Source: original research

The target audience was represented by respondents that has been selected from a database of Romanian digital agencies. The six-item questionnaire has been sent via a link to more than 60 digital agencies' representatives until we have validated 25 cases.

The most important approach in configurational analysis is data calibration, which involves the process of turning the values of all antecedent conditions on 5 point scales and outcome as well into fuzzy sets with values ranging from 0 to 1 (Table 1).

Table 1 - Calibration of scales

\begin{tabular}{|c|c|c|c|}
\hline \multicolumn{2}{|c|}{ Scale point } & Fuzzy-set value & Membership \\
\hline Strongly agree/Very probably & 5 & 1 & Fully in \\
\hline Agree/Probably & 4 & 0.75 & $\begin{array}{c}\text { Cross-over (neither in } \\
\text { nor out) }\end{array}$ \\
\hline $\begin{array}{c}\text { Neutral opinion/ } \\
\text { Possibly }\end{array}$ & 3 & 0.5 & More out than in \\
\hline $\begin{array}{c}\text { Disagree/ } \\
\text { Probably not }\end{array}$ & 2 & 0.25 & Fully out \\
\hline $\begin{array}{c}\text { Strongly disagree/Definitely } \\
\text { not }\end{array}$ & 1 & 0.1 & $($ Source: \\
\hline
\end{tabular}

(Source: adapted from Ragin, 2008)

We have considered three qualitative anchors for the calibration: an anchor to define full membership, an anchor to define an almost complete lack of membership, and a crossover point, 
to check the matching to full-set membership, full-set non-membership and intermediate-set membership, revealing the degree that a case is part or not of a set.

Building on complexity theory and the principle of equifinality, a result may be equally explained by alternative sets of causal conditions (Fiss, 2007). The antecedent conditions employed in this research should be combined in sufficient configurations to explain the outcome (Woodside, 2014).

The definition of the fuzzy-set values for the antecedent conditions (visibility, advertising, incentives, behaviour and predictions) was in line with the same rule for calibration of the outcome (respondents' intention to test AI Media platform), emphasizing a comprehensive overview of the cases in the selected sample of potential users of the platform. Table 2 reflects a new variable: antecedents, as the result of computing the fuzzy-set values of the five conditions from the conceptual model, using fsQCA software:

antecedents =fuzzyand(Visibility,Advertising,Incentives,Behaviour,Predictions)

\section{Table 2 - Calibration of antecedent conditions}

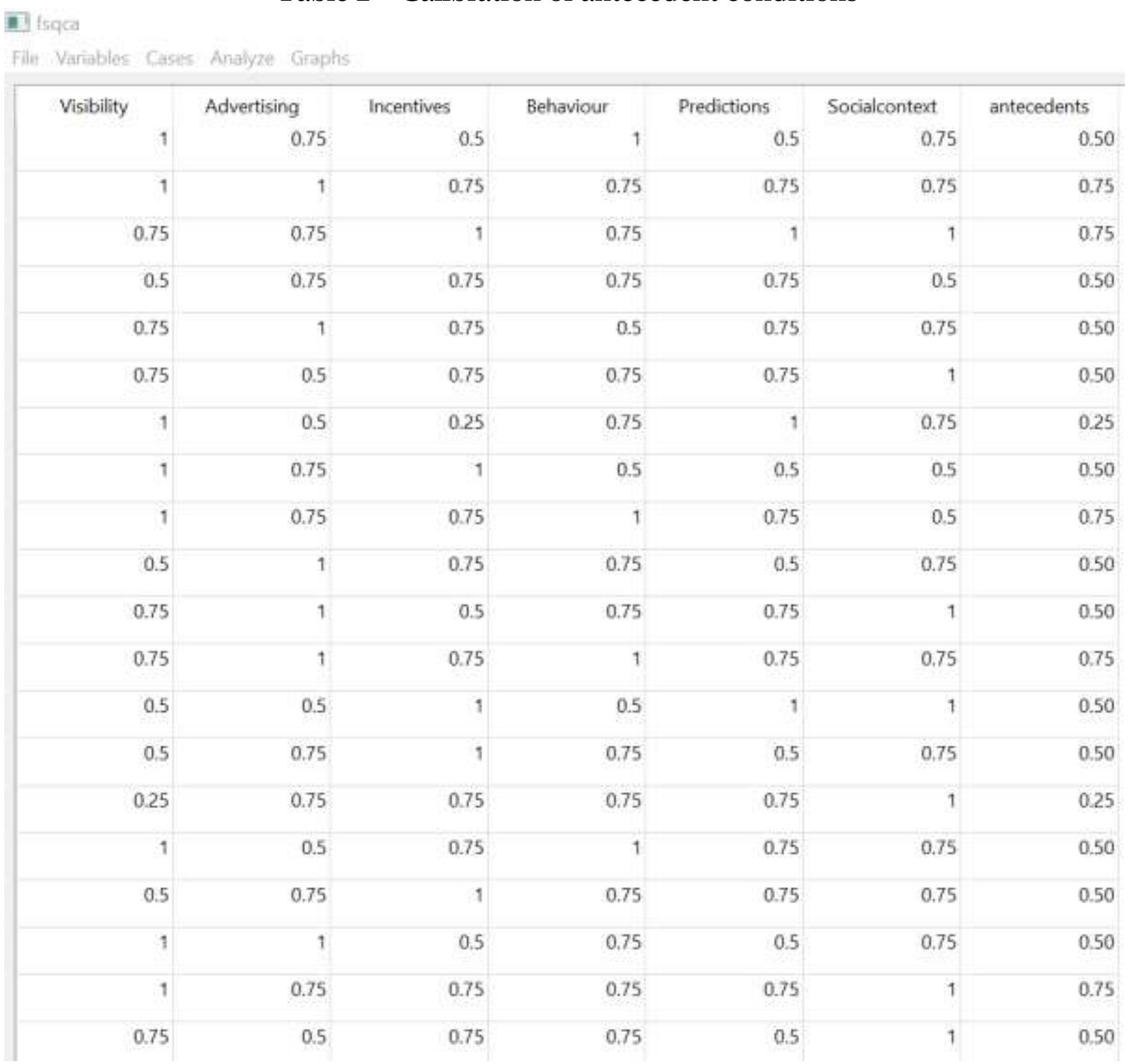

Source: own contribution, based on a fsQCA software output 
The gaps between the fuzzy-set values in the sample were consistent with respondents' different perceptions of geotagged image and social context recognition capabilities in the case of AI Media platform.

\section{Findings}

The first step of analysis aims at determining the consistency and coverage scores on fuzzy-set XY plots (Figure 2). The graphical representation of the results suggest that antecedent conditions are sufficient for the outcome in the purposely selected sample, as reflected by the positioning of nineteen cases above the diagonal in the XY plots, a single case below the diagonal in the XY plot and five cases lying on the diagonal.

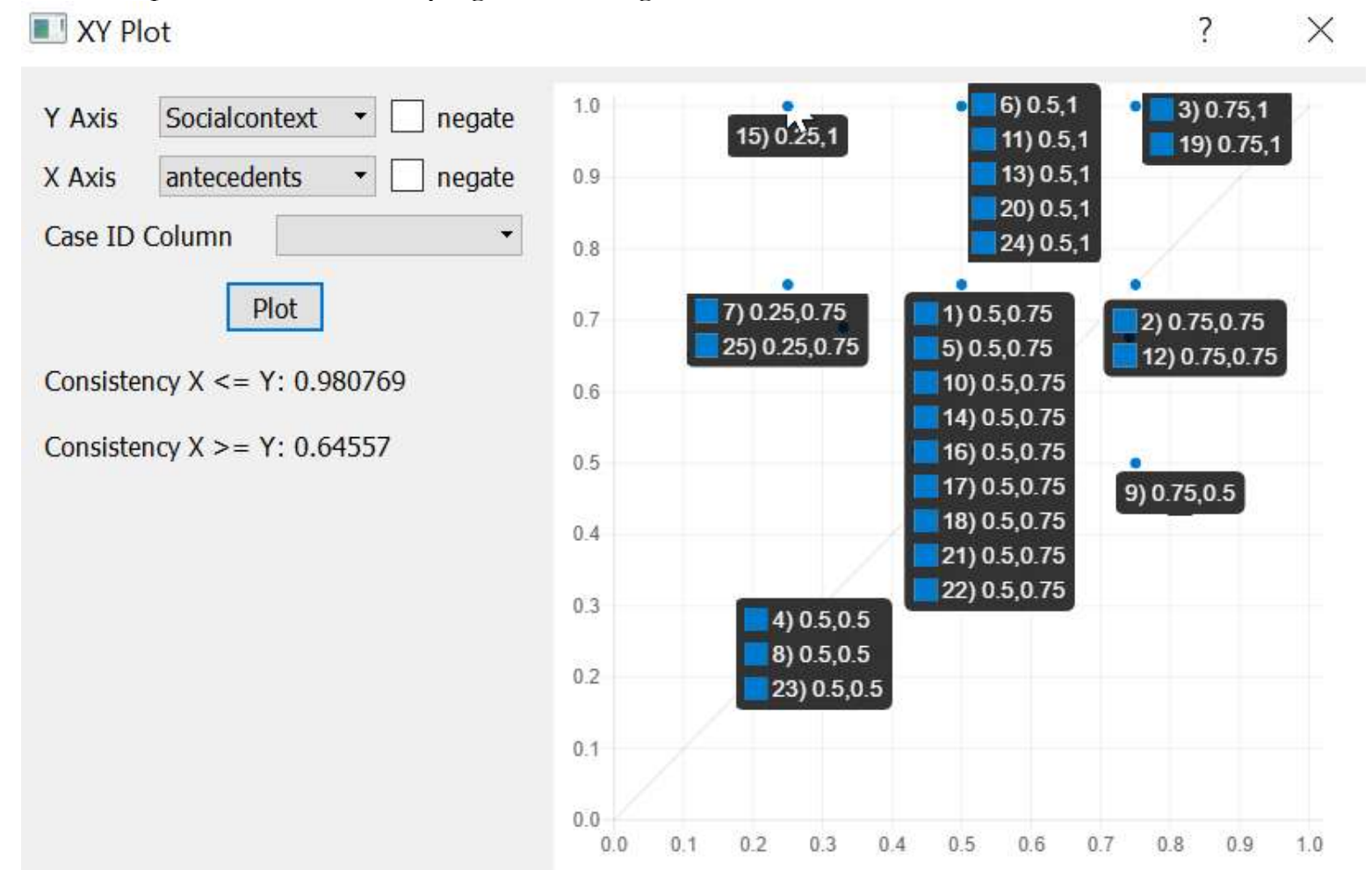

Figure 3 - Graphical distribution of fuzzy-sets within the sample

Source: own contribution, based on a fsQCA software output

For the selected sample, the consistency score (0.98), and the coverage score $(0.64)$ denote that the distribution of fuzzy sets is largely consistent with the assertion that the five antecedent conditions represent a subset of the outcome (respondents' intention to test AI Media platform). Antecedent conditions' coverage of the outcome is $64 \%$.

The complex solution provided by the Quine-McCluskey algorithm outlines that two configurations of antecedent conditions affect the outcome and two other affect the negated outcome. Their combination represents a successful recipe for a higher interest to test the features of the AI Media platform. Gaining visibility through brand related photo or video geotagged on social media is perceived by the respondents as more influential than the other four advantages proposed by the AI Media platform, as it was found in both configurations affecting the outcome.

The equifinality principle (multiple paths to a desired outcome may coexist, according to Fiss, 2007) is proved, as multiple paths of antecedent conditions lead to the outcome (Table 3). 
Table 3: Causal recipes for intention to test the future AI-based software within the Romanian fuzzy-set sub-sample

\begin{tabular}{|c|c|c|c|c|c|}
\hline \multirow{2}{*}{ Configuration } & \multicolumn{2}{|c|}{$\begin{array}{l}\text { High intention to test the } \\
\text { future AI-based platform }\end{array}$} & \multicolumn{3}{|c|}{$\begin{array}{l}\text { Low/no intention to test the } \\
\text { future AI-based platform }\end{array}$} \\
\hline & 1 & 2 & 1 & 2 & 3 \\
\hline $\begin{array}{l}\text { Gaining visibility through brand } \\
\text { related photo or video } \\
\text { geotagged on social media }\end{array}$ & $\bullet$ & $\bullet$ & & & • \\
\hline $\begin{array}{l}\text { Free advertising through tagging } \\
\text { a location while people add an } \\
\text { image containing a brand's logo }\end{array}$ & $\bullet$ & $\otimes$ & $\otimes$ & $\otimes$ & \\
\hline $\begin{array}{l}\text { Incentives given to geotagged } \\
\text { images uploaded on social } \\
\text { networks and }\end{array}$ & $\otimes$ & & $\otimes$ & & \\
\hline $\begin{array}{l}\text { Richer behavioural data } \\
\text { provided by geotagged image } \\
\text { recognition features }\end{array}$ & & $\bullet$ & & $\otimes$ & $\otimes$ \\
\hline $\begin{array}{c}\text { Capitalizing on location-based } \\
\text { image recognition to predict } \\
\text { consumer behaviour and } \\
\text { improve services }\end{array}$ & $\bullet$ & $\otimes$ & $\otimes$ & & \\
\hline Consistency & 0.98 & 0.96 & 0.48 & 0.45 & 0.52 \\
\hline Raw coverage & 0.65 & 0.63 & 0.54 & 0.52 & 0.46 \\
\hline Unique coverage & 0.65 & 0.63 & 0.52 & 0.48 & 0.44 \\
\hline Overall solution consistency & & & & 0.46 & \\
\hline Overall solution coverage & & & & 0.52 & \\
\hline
\end{tabular}

The black circles $(\bullet)$ reveal the presence of a condition, while crossed-out circles $(\otimes)$ show its absence (or low impact), according to Pappas et al., 2016. Blank spaces show the "do not care" situation. All values related to consistency scores are greater from the recommended threshold of 0.75 (Ragin, 2008) in the case of the outcome., while, in the case of the negated outcome, the consistency scores are lower from the recommended threshold of 0.75 .

\section{Conclusions}

Following the results of this study, software developers from the FutureWeb research team gain meaningful insights on how the clients of the future AI Media platform perceive geotagged image and social context recognition capabilities. The five features, considered as antecedent conditions in the fsQCA logic, will help them improve their work, by focusing on the expectations of the final users of the platform. Furthermore, we identified alternative paths that explain high intentions to test the AI Media platform. 
The AI platform is now ready for the inclusion the beta test phase, with the support of IT professionals from the research team; the marketing professionals from the same research team are responsible for adressing the valuable feedback from potential users, regarding their expectations on the geotagged image and social context recognition capabilities.

\section{Acknowledgments}

The research was conducted within the framework of the project FutureWeb, funded by Romanian Ministry of Research and Innovation, CCCDI - UEFISCDI, project number PN-IIIP1-1.2-PCCDI-2017-0800 / 86PCCDI/2018, within PNCDI III.

\section{References}

1. Choi, J., Thomee, B., Friedland, G., Cao, L., Ni, K., Borth, D., ... \& Poland, D. (2014, November). The placing task: A large-scale geo-estimation challenge for social-media videos and images. In Proceedings of the 3rd ACM Multimedia Workshop on Geotagging and Its Applications in Multimedia (pp. 27-31). ACM.

2. Fiss, P. C. (2007). "A set-theoretic approach to organizational configurations". Academy of management review, 32(4), 1180-1198.

3. Harris, P. B., Mitchell, S. K., \& Mulroy, M. J. (2014). U.S. Patent No. 8,862,589. Washington, DC: U.S. Patent and Trademark. Office.

4. Hays, J., \& Efros, A. A. (2008, June). IM2GPS: estimating geographic information from a single image. In 2008 IEEE conference on computer vision and pattern recognition (pp. 1-8). IEEE.

5. Ivanov, I., Vajda, P., Lee, J. S., Goldmann, L., \& Ebrabimi, T. (2012). Geotag propagation in social networks based on user trust model. Multimedia Tools and Applications, 56(1), 155-177.

6. Knopp, J., Sivic, J., \& Pajdla, T. (2010, September). Avoiding confusing features in place recognition. In European Conference on Computer Vision (pp. 748-761). Springer, Berlin, Heidelberg.

7. Lee, S., Zhang, H., \& Crandall, D. J. (2015, January). Predicting geo-informative attributes in large-scale image collections using convolutional neural networks. In 2015 IEEE Winter Conference on Applications of Computer Vision (pp. 550-557). IEEE.

8. Luo, J., Boutell, M., \& Brown, C. (2006). Pictures are not taken in a vacuum-an overview of exploiting context for semantic scene content understanding. IEEE Signal Processing Magazine, 23(2), 101-114.

9. Luo, J., Joshi, D., Yu, J., \& Gallagher, A. (2011). Geotagging in multimedia and computer vision-a survey. Multimedia Tools and Applications, 51(1), 187-211.

10. Pappas, I. O., Kourouthanassis, P. E., Giannakos, M. N., and Chrissikopoulos, V. (2016). "Explaining online shopping behavior with fsQCA: The role of cognitive and affective perceptions". Journal of Business Research, 69(2), 794-803

11. Ragin, C. C. (2008). Redesigning social inquiry: Furzy sets and beyond (Vol. 240): Wiley Online

12. Tsai, C. M., Qamra, A., Chang, E. Y., \&Wang, Y. F. (2005, July). Extent: Inferring image metadata from context and content. In 2005 IEEE International Conference on Multimedia and Expo (pp. 12701273). IEEE.

13. Yaegashi, K., \& Yanai, K. (2010, November). Geotagged image recognition by combining three different kinds of geolocation features. In Asian Conference on Computer Vision (pp. 360-373). Springer, Berlin, Heidelberg.

14. Yuan, J., Luo, J., Kautr, H., \& Wu, Y. (2008, October). Mining GPS traces and visual words for event classification. In Proceedings of the 1 st ACM international conference on Multimedia information retrieval (pp. 2-9). ACM.

15. Woodside, A. G. (2014). "Embrace perform $\bullet$ model: Complexity theory, contrarian case analysis, and multiple realities". Journal of Business Research, 67(12), 2495-2503 


\section{Appendix}

\section{Questionnaire}

1. Having your brand related photo or video geotagged on social media is a great way to gain visibility. (Strongly agree ... Strongly disagree)

2. When people tag a location while they add an image containing your brand's logo, it's practically free advertising for your company in social media. (Strongly agree ... Strongly disagree)

3. Since your brand's engagement is high when the posts of your fans, who upload content related to your brand, are geotagged, you should offer them incentives. (Strongly agree ... Strongly disagree)

4. Using geotagged image recognition features, your company's Social Media Marketing strategy will benefit from richer behavioural data. (Strongly agree ... Strongly disagree)

5. One of the strengths of your company's Social Media Marketing strategy is to capitalize on location-based image recognition to predict consumer behaviour and improve your services. (Strongly agree ... Strongly disagree)

6. Your company is looking forward testing an AI-based software, capable to detect and classify image and video content posted in social networks in peculiar social contexts. (Very probably ... Definitely not) 